\title{
PTOLEMY SPACES WITH STRONG INVERSIONS
}

\author{
A. SMIRNOV
}

\begin{abstract}
It is proved that a compact Ptolemy space with many strong inversions that contains a Ptolemy circle is Möbius equivalent to an extended Euclidean space.
\end{abstract}

\section{$\S 1$. INTRODUCTION}

This work is motivated by the papers BS1 and BS2 of S. Buyalo and V. Schroeder, which give a Möbius characterization of the boundary at infinity of the rank one symmetric spaces of noncompact type. That characterization employs the notion of a space inversion with respect to distinct points $\omega, \omega^{\prime} \in X$ and a metric sphere $S \subset X$ between $\omega, \omega^{\prime}$. By definition, such an inversion is a Möbius automorphism $\varphi=\varphi_{\omega, \omega^{\prime}, S}: X \rightarrow X$ with the following properties:

(1) $\varphi$ is an involution without fixed points, $\varphi^{2}=$ id;

(2) $\varphi(\omega)=\omega^{\prime}$ (and thus $\left.\varphi\left(\omega^{\prime}\right)=\omega\right)$;

(3) $\varphi$ preserves $S, \varphi(S)=S$;

(4) $\varphi(\sigma)=\sigma$ for any Ptolemy circle $\sigma \subset X$ through $\omega, \omega^{\prime}$.

Recall that, however, a classical inversion of the Euclidean space $\mathbb{R}^{n}$ with respect to a sphere $S \subset \mathbb{R}^{n}$ fixes $S$ pointwise. In this paper we impose a stronger condition on an s-inversion, assuming that $\varphi$ preserves $S$ pointwise, $\varphi(x)=x$ for every $x \in S$; such an inversion will be called a strong s-inversion. We study Ptolemy spaces with the following two properties.

(E) Existence: there is at least one Ptolemy circle in $X$.

(sI) Strong inversions: for any distinct $\omega, \omega^{\prime} \in X$ and any metric sphere $S \subset X$ between $\omega, \omega^{\prime}$ there is a strong space inversion $\varphi_{\omega, \omega^{\prime}, S}: X \rightarrow X$ with respect to $\omega, \omega^{\prime}$ and $S$.

Our main goal is the proof of the following theorem.

Theorem 1. Let $X$ be a compact Ptolemy space with properties (E) and (sI). Then $X$ is Möbius equivalent to the extended Euclidean space $\widehat{\mathbb{R}}^{n}=\mathbb{R}^{n} \cup\{\infty\}$ for some $n \geq 1$.

Another Möbius characterization of $\widehat{\mathbb{R}}^{n}$ was obtained in [FS] : a compact Ptolemy space $X$ is Möbius equivalent to $\widehat{\mathbb{R}}^{n}$ if and only if any three points in $X$ lie on a Ptolemy circle.

Despite the differences in the definition of s-inversions and strong s-inversions, some properties of the spaces under study pertain to both cases. Thus, the definitions of the homotheties and shifts, as well as Lemmas 4, 5, 6] were originally presented in [BS1]. Significant differences between the two classes arise when we consider the symmetry with respect to a horosphere. In general, if we only assume the existence of s-inversions, there is no reason for such a symmetry to exist.

2010 Mathematics Subject Classification. Primary 53C70.

Key words and phrases. Ptolemy space, Möbius structure.

Supported by RFBR (grant no. 11-01-00302-a). 


\section{$\S 2$. BASIC DEFINITIONS}

2.1. Möbius structures. In this section we follow the definitions in BS1. Namely, fix a set $X$ and consider the extended metrics on $X$ for which the existence of an infinitely remote point $\omega \in X$ is allowed, that is, $d(x, \omega)=\infty$ for all $x \in X, x \neq \omega$. We always assume that such a point is unique if it exists, and that $d(\omega, \omega)=0$.

A quadruple $Q=(x, y, z, u)$ of points in a set $X$ is said to be admissible if each entry occurs at most two times in $Q$. Two metrics $d, d^{\prime}$ on $X$ are Möbius equivalent if for any admissible quadruple $Q=(x, y, z, u) \subset X$ the respective cross-ratio triples coincide, $\operatorname{crt}_{d}(Q)=\operatorname{crt}_{d^{\prime}}(Q)$, where

$$
\operatorname{crt}_{d}(Q)=(d(x, y) d(z, u): d(x, z) d(y, u): d(x, u) d(y, z)) \in \mathbb{R} P^{2} .
$$

If $\infty$ occurs once in $Q$, say $u=\infty$, then $\operatorname{crt}_{d}(x, y, z, \infty)=(d(x, y): d(x, z): d(y, z))$. If $\infty$ occurs twice, say $z=u=\infty$, then $\operatorname{crt}_{d}(x, y, \infty, \infty)=(0: 1: 1)$.

A Möbius structure on a set $X$ is a class $\mathcal{M}=\mathcal{M}(X)$ of metrics on $X$ that are pairwise Möbius equivalent.

The topology on $(X, d)$ is that with the base consisting of all open distance balls $B_{r}(x)$ around points in $x \in X_{\omega}$ and the complements $X \backslash D$ of all closed distance balls $D=\bar{B}_{r}(x)$. Möbius equivalent metrics give rise to one and the same topology on $X$. When a Möbius structure $\mathcal{M}$ on $X$ is fixed, we say that $(X, \mathcal{M})$ or simply $X$ is a Möbius space.

A map $f: X \rightarrow X^{\prime}$ between two Möbius spaces is said to be Möbius if $f$ is injective and for all admissible quadruples $Q \subset X$ we have

$$
\operatorname{crt}(f(Q))=\operatorname{crt}(Q)
$$

where the cross-ratio triples are taken with respect to some (and hence an arbitrary) metric of the Möbius structures of $X, X^{\prime}$. Möbius maps are continuous. If a Möbius map $f: X \rightarrow X^{\prime}$ is bijective, then $f^{-1}$ is Möbius, $f$ is a homeomorphism, and the Möbius spaces $X, X^{\prime}$ are said to be Möbius equivalent.

We note that if two Möbius equivalent metrics have the same infinitely remote point, then they are homothetic, see, e.g., [BS1, FS].

A classical example of a Möbius space is the extended $\widehat{\mathbb{R}}^{n}=\mathbb{R}^{n} \cup \infty=S^{n}, n \geq 1$, where the Möbius structure is generated by some extended Euclidean metric on $\widehat{\mathbb{R}}^{n}$, and $\mathbb{R}^{n} \cup \infty$ is identified with the unit sphere $S^{n} \subset \mathbb{R}^{n+1}$ via the stereographic projection.

2.2. Ptolemy spaces. A Möbius space $X$ is called a Ptolemy space if it satisfies the Ptolemy property, that is, for all admissible quadruples $Q \subset X$ the entries of the respective cross-ratio triple $\operatorname{crt}(Q) \in \mathbb{R} P^{2}$ satisfy the triangle inequality.

The Ptolemy property is equivalent to the fact that the Möbius structure $\mathcal{M}$ of $X$ is invariant under the metric inversions, or in other words, $\mathcal{M}$ is Ptolemy if and only if for all $z \in X$ there exists a metric $d_{z} \in \mathcal{M}$ with infinitely remote point $z$.

Recall that the metric inversion (or m-inversion for brevity) of a metric $d \in \mathcal{M}(X)$ with respect to $z \in X \backslash \omega$ ( $\omega$ is infinitely remote for $d$ ) of radius $r>0$ is the function $d_{z}(x, y)=\frac{r^{2} d(x, y)}{d(z, x) d(z, y)}$ for all $x, y \in X$ distinct from $z, d_{z}(x, z)=\infty$ for all $x \in X \backslash\{z\}$ and $d_{z}(z, z)=0$.

The classical example of a Ptolemy space is $\widehat{\mathbb{R}}^{n}$ with a standard Möbius structure.

An interesting basic fact about Ptolemy spaces is the following Schoenberg theorem.

Theorem 2 ([Sch $)$. If a real normed vector space is a Ptolemy space, then it is an inner product space. 
A Ptolemy circle in a Ptolemy space $X$ is a subset $\sigma \subset X$ homeomorphic to $S^{1}$ and such that for every quadruple $(x, y, z, u) \in \sigma$ of distinct points we have

$$
d(x, z) d(y, u)=d(x, y) d(z, u)+d(x, u) d(y, z)
$$

for some (and hence, an arbitrary) metric $d$ of the Möbius structure, where it is assumed that the pair $(x, z)$ separates the pair $(y, u)$, i.e., $y$ and $u$ are in different components of $\sigma \backslash\{x, z\}$.

Given $\omega \in X$, we use the notation $X_{\omega}=X \backslash \omega$ and always assume that a metric of the Möbius structure on $X_{\omega}$ is fixed. Note that every Ptolemy circle $\sigma \subset X$ that passes through $\omega$ is isometric to a geodesic line in $X_{\omega}$. Such a line $\ell=\sigma_{\omega}$ is called a Ptolemy line.

2.3. Space inversions. Given distinct $\omega, \omega^{\prime} \in X$, we say that a subset $S \subset X$ is a metric sphere between $\omega, \omega^{\prime}$ if

$$
S=\{x \in X: d(x, \omega)=r\}=S_{r}^{d}(\omega)
$$

for some metric $d \in \mathcal{M}$ with infinitely remote point $\omega^{\prime}$ and some $r>0$. Any two such metrics $d, d^{\prime} \in \mathcal{M}$ are proportional to each other, $d^{\prime}=\lambda d$ for some $\lambda>0$. Then $S_{r}^{d}(\omega)=$ $S_{\lambda r}^{d^{\prime}}(\omega)$. Moreover, this notion is symmetric relative to $\omega, \omega^{\prime}$, because any metric $d^{\prime} \in \mathcal{M}$ with infinitely remote point $\omega$ is proportional to the m-inversion of $d$ with respect to $\omega$, and we may assume that $d^{\prime}$ is an m-inversion itself. Then $S=\left\{x \in X: d^{\prime}\left(x, \omega^{\prime}\right)=1 / r\right\}$.

We define a strong space inversion, or s-inversion for brevity, with respect to distinct $\omega, \omega^{\prime} \in X$ and a metric sphere $S \subset X$ between $\omega, \omega^{\prime}$ as a Möbius automorphism $\varphi=\varphi_{\omega, \omega^{\prime}, S}: X \rightarrow X$ such that

(1) $\varphi$ is an involution, $\varphi^{2}=\mathrm{id}$;

(2) $\varphi(\omega)=\omega^{\prime}$ (and thus, $\left.\varphi\left(\omega^{\prime}\right)=\omega\right)$;

(3) $\varphi$ preserves $S$ pointwise, $\varphi(x)=x$ for every $x \in S$

(4) $\varphi(\sigma)=\sigma$ for any Ptolemy circle $\sigma \subset X$ through $\omega, \omega^{\prime}$.

Let $\omega \in X$. Fix $o \in X_{\omega}$ and consider a metric sphere $S=S_{r}(o)$ between $o$ and $\omega$. Let $\varphi$ be an s-inversion with respect to $o, \omega$ and $S$. Now we prove two technical lemmas.

Lemma 1. Let $x \in X_{\omega}$. Then $|o x| \cdot|o \varphi(x)|=r^{2}$.

Proof. Let $y \in S$. Then

$$
\begin{aligned}
\operatorname{crt}(x, y, o, \omega)=(|x y|:|x o|:|y o|) & =\operatorname{crt}(\varphi(x), \varphi(y), \varphi(o), \varphi(\omega)) \\
& =\operatorname{crt}(\varphi(x), y, \omega, o)=(|\varphi(x) y|:|y o|:|\varphi(x) o|) .
\end{aligned}
$$

It follows that $|\varphi(x) o| /|y o|=|y o| /|x o|$ and $|o x| \cdot|o \varphi(x)|=r^{2}$.

Lemma 2. Let $x, y \in X_{\omega}$. Then $|\varphi(x) \varphi(y)|=r^{2} \cdot \frac{|x y|}{|o x| \cdot|o y|}$.

Proof. Note that

$\operatorname{crt}(x, y, o, \omega)=(|x y|:|x o|:|y o|)=\operatorname{crt}(\varphi(x), \varphi(y), \omega, o)=(|\varphi(x) \varphi(y)|:|\varphi(y) o|:|\varphi(x) o|)$.

Consequently, $|\varphi(x) \varphi(y)| /|\varphi(x) o|=|x y| /|y o|$. By Lemma 1, $|\varphi(x) o|=r^{2} /|x o|$. Then $|\varphi(x) \varphi(y)|=|\varphi(x) o| \cdot \frac{|x y|}{|y o|}=r^{2} \frac{|x y|}{|o x| \cdot|o y|} \cdot$

We say that a Möbius space $X$ possesses property (E) if there is a Ptolemy circle in $X$. And we also say that a Möbius space $X$ possesses property (sI) if for any distinct $\omega$, $\omega^{\prime} \in X$ and a metric sphere $S \subset X$ between $\omega, \omega^{\prime}$ there is an s-inversion $\varphi_{\omega, \omega^{\prime}, S}: X \rightarrow X$ with respect to $\omega, \omega^{\prime}$, and $S$.

From now on, we assume that $X$ is a compact Ptolemy space with properties (E) and (sI). 


\section{$\S 3$. Homotheties AND ShIFTs}

3.1. Homotheties. Fix $\omega \in X$. Let $o \in X_{\omega}, \lambda>0$. Consider $r_{1}, r_{2}>0$ such that $\lambda=r_{2}^{2} / r_{1}^{2}$. Let $S_{1}=S_{r_{1}}(o), S_{2}=S_{r_{2}}(o) \subset X_{\omega}$ be metric spheres between $o, \omega$. Denote by $\varphi_{1}, \varphi_{2}$ s-inversions relative to $o, \omega, S_{1}$ and $o, \omega, S_{2}$, respectively.

We define a homothety with the center $o$ and the coefficient $\lambda$ as a Möbius authomorphism $h: X \rightarrow X$ such that $h=\varphi_{2} \circ \varphi_{1}$.

The next properties follow from the definition of an s-inversion and from Lemma 2

(1) $h(o)=o, h(\omega)=\omega$.

(2) $h(\sigma)=\sigma$ for any Ptolemy circle $\sigma \subset X$ through $o, \omega$.

(3) $|h(x) h(y)|=\lambda|x y|$ for all $x, y \in X_{\omega}$.

(4) For each $o \in X_{\omega}$ and each $\lambda>0$, there exists a homothety with the center $o$ and the coefficient $\lambda$.

We denote a homothety with center $o$ and coefficient $\lambda$ by $h_{\lambda, o}$.

Proposition 1. Let $\omega, \omega^{\prime} \in X$, let $\sigma$ be a Ptolemy circle through $\omega, \omega^{\prime}$, and let $\Gamma \subset \sigma$ be a connected component of $\sigma \backslash\left\{\omega, \omega^{\prime}\right\}$. Consider $x, x^{\prime} \in \Gamma$. Then there exists a homothety $h$ with the center $\omega^{\prime}$ such that $h(x)=x^{\prime}$.

Proof. Consider a metric space $X_{\omega}$. Since $\omega \in \sigma, \Gamma$ is a geodesic ray starting at $\omega^{\prime}$. Define $\lambda$ by $\left|\omega^{\prime} x^{\prime}\right|=\lambda\left|\omega^{\prime} x\right|$. Then $h(x)=x^{\prime}$ for $h=h_{\lambda, \omega^{\prime}}$.

Corollary 1. Any two distinct Ptolemy circles in a Ptolemy space with properties (E) and (sI) have at most two points in common.

Proof. Let $\sigma, \sigma^{\prime} \subset X$ be intersecting Ptolemy circles with $\omega \in \sigma \cap \sigma^{\prime}$. Consider the metric space $X_{\omega}$. Arguing by contradiction, suppose that there exist $x, x^{\prime} \in\left(\sigma \cap \sigma^{\prime}\right) \backslash\{\omega\}$. Let $\Gamma$ be a connected component of $\sigma \backslash\{x, \omega\}$ such that $x^{\prime} \in \Gamma$. Also, let $\Gamma^{\prime}$ be a connected component of $\sigma^{\prime} \backslash\{x, \omega\}$ such that $x^{\prime} \in \Gamma^{\prime}$. Note that if $x^{\prime \prime} \in \Gamma$ and $\lambda=\left|x x^{\prime \prime}\right| /\left|x x^{\prime}\right|$, then for a homothety $h=h_{\lambda, x}$ we have $h\left(x^{\prime}\right)=x^{\prime \prime}$. Then $x^{\prime \prime} \in \Gamma^{\prime}$ and $\Gamma \subset \Gamma^{\prime}$. Similarly, $\Gamma^{\prime} \subset \Gamma$, whence $\Gamma \equiv \Gamma^{\prime}$. In the same way, if $\Gamma_{1}$ is a connected component of $\sigma \backslash\left\{x^{\prime}, \omega\right\}$ such that $x \in \Gamma_{1}$, and $\Gamma_{1}^{\prime}$ is a connected component of $\sigma^{\prime} \backslash\left\{x^{\prime}, \omega\right\}$ such that $x \in \Gamma_{1}^{\prime}$, we can check that $\Gamma_{1}=\Gamma_{1}^{\prime}$. It follows that $\sigma=\sigma^{\prime}$.

3.2. Shifts. Note that $X$ is Hausdorff and compact. If we fix a nonprincipal ultrafilter $\theta$ on the set of natural numbers $\mathbb{N}$, then for each sequence $x_{n} \in X$ there exists a unique $x \in X \operatorname{such} x=\lim _{\theta} x_{n}$. Moreover, $\left|\lim _{\theta}\left(x_{n}\right) \lim _{\theta}\left(y_{n}\right)\right|=\lim _{\theta}\left|x_{n} y_{n}\right|$ for all sequences $x_{n}, y_{n} \in X$.

In this section we need the following well-known fact, see, e.g., BS1, Lemma 6.7].

Lemma 3. Assume that, for a nondegenerate triple $T=(x, y, z) \subset X$ and a sequence $\varphi_{i} \in \operatorname{Mob} X$, the sequence $T_{i}=\varphi_{i}(T) \theta$-converges to a nondegenerate triple $T^{\prime}=\left(x^{\prime}, y^{\prime}, z^{\prime}\right) \subset X$. Then there exists $\varphi=\lim _{\theta} \varphi_{i} \in \operatorname{Mob} X$ with $\varphi(T)=T^{\prime}$.

Fix $\omega \in X$ and let $x, x^{\prime} \in X_{\omega}$. Let $\lambda_{n}>0, n \in \mathbb{N}$, be a sequence that goes to zero. Consider the homothety $h_{n}$ with center $x$ and coefficient $\lambda_{n}^{-1}$ and the homothety $h_{n}^{\prime}$ with center $x^{\prime}$ and coefficient $\lambda_{n}$. We denote their composition $h_{n}^{\prime} \circ h_{n}$ by $\eta_{n}$. Note that $\eta_{n}$ is an isometry for each $n \in \mathbb{N}$. Then, by Lemma 3. $\eta=\lim _{\theta} \eta_{n}$ is a Möbius automorphism with $\eta(x)=x^{\prime}$ and $\eta(\omega)=\omega$. Moreover $\eta: X_{\omega} \rightarrow X_{\omega}$ is an isometry. We denote it by $\eta_{x x^{\prime}}$ and call a shift from $x$ to $x^{\prime}$. For every $x, x^{\prime} \in X_{\omega}$, there exists a shift from $x$ to $x^{\prime}$. 


\section{$\S 4$. Foliations by parallel Lines}

Since each Ptolemy line $\ell \subset X_{\omega}$ is isometric to $\mathbb{R}$, for every $x_{0} \in \ell$ the Busemann functions $b_{\ell, x_{0}}^{ \pm}: X_{\omega} \rightarrow \mathbb{R}$ are well defined by the formula

$$
b_{\ell, x_{0}}^{ \pm}(x)=\lim _{t \rightarrow \pm \infty}|x c(t)|-\left|x_{0} c(t)\right|
$$

where $c(t): \mathbb{R} \rightarrow \ell$ is a unit speed parametrization.

Two Ptolemy lines $\ell, \ell^{\prime} \subset X_{\omega}$ are said to be Busemann parallel if $\ell, \ell^{\prime}$ share Busemann functions, that is, any Busemann function associated with $\ell$ is also a Busemann function associated with $\ell^{\prime}$ and vice versa.

The following lemmas were proved in BS1], and the proofs go without changes in our case.

Lemma 4 ([BS1, Lemma 4.11]). Let $\ell, \ell^{\prime} \subset X_{\omega}$ be Ptolemy lines with a common point $o \in l \cap \ell^{\prime}$ and let $b: X_{\omega} \rightarrow \mathbb{R}$ be a Busemann function of $\ell$ with $b(o)=0$. Assume that $b \circ c(t)=-t=b \circ c^{\prime}(t)$ for all $t \geq 0$ and for appropriate unit speed parametrizations $c, c^{\prime}: \mathbb{R} \rightarrow X_{\omega}$ of $\ell, \ell^{\prime}$ (respectively) with $c(0)=o=c^{\prime}(0)$. Then $l=l^{\prime}$. In particular, Busemann parallel Ptolemy lines coincide if they have a common point.

Lemma 5 ([BS1, Lemma 4.12]). Let $c, c^{\prime}: \mathbb{R} \rightarrow X_{\omega}$ be unit speed parametrizations of Ptolemy lines $\ell, \ell^{\prime} \subset X_{\omega}$, respectively. If $\left|c\left(t_{i}\right) c^{\prime}\left(t_{i}\right)\right| /\left|t_{i}\right| \rightarrow 0$ for some sequences $t_{i} \rightarrow \pm \infty$, then the lines $\ell, \ell^{\prime}$ are Busemann parallel.

Vice versa, if $\ell, \ell^{\prime} \subset X_{\omega}$ are Busemann parallel lines, then

$$
\lim _{t \rightarrow \infty}\left|c(t) c^{\prime}(t)\right| / t=0
$$

for their unit speed parametrizations $c, c^{\prime}: \mathbb{R} \rightarrow X_{\omega}$ chosen appropriately.

Lemma 6 ([BS1, Lemma 4.13]). A shift $\eta_{x x^{\prime}}$ moves any Ptolemy line $\ell$ through $x$ to a Busemann parallel Ptolemy line $\eta_{x x^{\prime}}(l)$ through $x^{\prime}$.

From Lemma 4 and Lemma 6 we immediately obtain the following claim.

Corollary 2. Given a Ptolemy line $\ell \subset X_{\omega}$, through any point $x \in X_{\omega}$ there is a unique Ptolemy line $l_{x}$ Busemann parallel to $\ell$.

\section{§5. Symmetries With Respect to horospheres}

In this section we construct a symmetry with respect to a horosphere.

Fix $\omega \in X$ and a Ptolemy line $\ell \subset X_{\omega}$, and let $c: \mathbb{R} \rightarrow X_{\omega}$ be a unit speed parametrization of $\ell$. For $t>0$, the metric sphere $S_{t}=\left\{x \in X_{\omega}:|x c(t)|=t\right\}$ passes through $z=c(0)$ and lies between $\omega$ and $c(t)$. By (sI), there is an s-inversion $\varphi_{t}=\varphi_{\omega, c(t), S_{t}}: X \rightarrow X$. By the compactness of $X$, the s-inversions $\varphi_{t}$ subconverge as $t \rightarrow \infty$ to a map $\varphi_{\infty}: X \rightarrow X$. Note that $\varphi_{\infty}(\omega)=\omega$ because $\varphi_{t}(c(t))=\omega$ and $c(t) \rightarrow \omega$ as $t \rightarrow \infty$.

Lemma 7. Let $x \in H_{z}$, where $H_{z} \subset X_{\omega}$ is the horosphere through $z \in \ell$ of the Busemann function $b^{+}(y)=\lim _{t \rightarrow \infty}(|y c(t)|-t), y \in X_{\omega}$. Then $\varphi_{\infty}(x)=x$.

Proof. Since $|z c(t)|=t$ for $t \geq 0$, we have $b^{+}(z)=0$. Let $\ell_{x}$ be a line through $x$ Busemann parallel to $\ell$, and let $c^{\prime}: \mathbb{R} \rightarrow X_{\omega}$ be its unit speed parametrization with $c^{\prime}(0)=x$ such that $b^{+}$is the Busemann function associated with the ray $c^{\prime}([0, \infty))$. Fix $\varepsilon>0$ and let $x^{\prime}=c^{\prime}(\varepsilon)$. Note that the function $\left|x^{\prime} c(t)\right|-t$ is monotone decreasing and tends to $b^{+}\left(x^{\prime}\right)=-\varepsilon$. On the other hand, $\left|x^{\prime} c(0)\right|>0$. This means that there exists $t>0$ such that $\left|x^{\prime} c(t)\right|-t=0$. Let $x_{t}=\varphi_{t}(x)$. Since $\varphi_{t}\left(x^{\prime}\right)=x^{\prime}$, by Lemma 2 we have

$$
\left|x_{t} x^{\prime}\right|=t^{2} \frac{\left|x x^{\prime}\right|}{|c(t) x| \cdot\left|c(t) x^{\prime}\right|}=\frac{t \varepsilon}{|c(t) x|} \text {. }
$$


Note that the function $|x c(t)|-t$ is monotone decreasing and tends to $b^{+}(x)=0$. This means that $|x c(t)| \geq t$ and $\left|x_{t} x^{\prime}\right| \leq \varepsilon$. It follows that $\left|x x_{t}\right| \leq\left|x x^{\prime}\right|+\left|x^{\prime} x_{t}\right| \leq 2 \varepsilon$. Choosing $\varepsilon \rightarrow 0$, we see that $\varphi_{t}(x) \rightarrow x$, and then $\varphi_{\infty}(x)=x$.

Now we show that $\varphi_{\infty}$ is an isometry of $X_{\omega}$ that, moreover, reflects the Ptolemy line $\ell$ in $z$. For each $x, y \in X_{\omega}$ and every sufficiently large $t>0$, by Lemma 2 we have

$$
\left|\varphi_{t}(x) \varphi_{t}(y)\right|=\frac{t^{2}|x y|}{|x c(t)||y c(t)|}
$$

and $|x c(t)|=t+b^{+}(x)+o(1),|y c(t)|=t+b^{+}(y)+o(1)$. Thus, $\left|\varphi_{\infty}(x) \varphi_{\infty}(y)\right|=|x y|$ for all $x, y \in X_{\omega}$, i.e., $\varphi_{\infty}$ is an isometry. It preserves the Ptolemy line $\ell$ because every $\varphi_{t}$ preserves the Ptolemy circle $\sigma=l \cup \omega$, and it reflects $\ell$ in $z$ because $\varphi_{\infty}(z)=z$ and every $\varphi_{t}$ is an s-inversion of $\sigma$.

\section{§6. Proof of Theorem 1}

6.1. Some metric relations. Recall that a Ptolemy space $X$ is said to be Busemann flat if for every Ptolemy circle $\sigma \subset X$ and every point $\omega \in \sigma$, we have $b^{+}+b^{-} \equiv$ const for opposite Busemann functions $b^{ \pm}: X_{\omega} \rightarrow \mathbb{R}$ associated with the Ptolemy line $\sigma_{\omega}$, see [BS1, §3.2].

Lemma 8. $X$ is Busemann flat.

Proof. Let $\ell \subset X_{\omega}$ be a Ptolemy line, and let $c: \mathbb{R} \rightarrow X_{\omega}$ be a unit speed parametrization of $\ell$. Consider the horosphere $H_{o}$ through $o=c(0)$ of the Busemann function $b^{+}(x)=$ $\lim _{t \rightarrow \infty}(|x c(t)|-t), x \in X_{\omega}$. Let $b^{-}(x)=\lim _{t \rightarrow \infty}(|x c(-t)|-t), x \in X_{\omega}$, and let $\varphi$ be the symmetry with respect to $H_{o}$. Note that if $x^{\prime}=\varphi(x)$, where $x, x^{\prime} \in X_{\omega}$, then $b^{+}(x)=b^{-}\left(x^{\prime}\right)$. Indeed,

$$
b^{-}\left(x^{\prime}\right)=\lim _{t \rightarrow \infty}\left(\left|x^{\prime} c(-t)\right|-t\right)=\lim _{t \rightarrow \infty}(|\varphi(x) \varphi(c(t))|-t)=\lim _{t \rightarrow \infty}(|x c(t)|-t)=b^{+}(x) .
$$

It follows that $b^{+}(z)=b^{-}(z)$ for every $z \in H_{o}$. Therefore, $H_{o}$ is also a horosphere of the Busemann function $b^{-}$, and then $b^{+}+b^{-} \equiv$ const.

Corollary 3. For each horosphere $H$ of the Busemann function $b^{+}$, the set $\varphi(H)$ is also a horosphere of the Busemann function $b^{+}$.

Lemma 9. Let $\ell, \ell^{\prime} \subset X_{\omega}$ be Busemann parallel lines, and let $\varphi: X_{\omega} \rightarrow X_{\omega}$ be the symmetry that reflects $\ell$ at $o \in \ell$. Then $\varphi$ reflects $\ell^{\prime}$ at $o^{\prime}=H_{o} \cap \ell^{\prime}$, where $H_{o}$ is the horosphere of $\ell$ through $o$.

Proof. $H_{o}$ is the fixed point set of $\varphi$ and $\varphi\left(\ell^{\prime}\right)$ is Busemann parallel to $\varphi(\ell)=\ell$. Thus, by Lemma 4 , $\varphi\left(\ell^{\prime}\right)=\ell^{\prime}$.

Lemma 10. Let $\ell, \ell^{\prime}$ be Busemann parallel lines in $X_{\omega}$, and let $x, y \in \ell, x^{\prime}, y^{\prime} \in \ell^{\prime}$ be such that $b(x)=b\left(x^{\prime}\right), b(y)=b\left(y^{\prime}\right)$, where $b$ is a common Busemann function of $\ell$ and $\ell^{\prime}$. Then $|x y|=\left|x^{\prime} y^{\prime}\right|,\left|x x^{\prime}\right|=\left|y y^{\prime}\right|,\left|x y^{\prime}\right|=\left|y x^{\prime}\right|$, and $\left|x^{\prime} y\right| \geq\left|x x^{\prime}\right|$.

Proof. The first identity is obvious because

$$
|x y|=|b(x)-b(y)|=\left|b\left(x^{\prime}\right)-b\left(y^{\prime}\right)\right|=\left|x^{\prime} y^{\prime}\right| .
$$

To prove the other two identities, consider the midpoint $z \in \ell$ between $x, y$, that is, $|x z|=|z y|$. Let $H_{x}, H_{y}, H_{z}$ be horospheres of $b$ through $x, y, z$ (respectively), and let $\varphi$ be the symmetry with respect to $H_{z}$ such that $\varphi(\ell)=\ell$. Note that $\varphi(x)=y$ and $\varphi\left(\ell^{\prime}\right)=\ell^{\prime}$. It follows that $\varphi\left(H_{x}\right)=H_{y}$. Moreover, $\varphi\left(x^{\prime}\right)=y^{\prime}$ and $\varphi\left(y^{\prime}\right)=x^{\prime}$. Then we have $\left|x x^{\prime}\right|=\left|y y^{\prime}\right|$ and $\left|x y^{\prime}\right|=\left|y x^{\prime}\right|$. 
Applying the Ptolemy inequality $|x y| \cdot\left|x^{\prime} y^{\prime}\right|+\left|x x^{\prime}\right| \cdot\left|y y^{\prime}\right| \geq\left|x y^{\prime}\right| \cdot\left|y x^{\prime}\right|$ to the quadruple $\left(x, x^{\prime}, y, y^{\prime}\right)$, we have

$$
|x y|^{2}+\left|x x^{\prime}\right|^{2} \geq\left|y x^{\prime}\right|^{2}
$$

On the other hand, if $y^{\prime \prime}$ is symmetric to $y$ with respect to $H_{x}$, then $\left|x y^{\prime \prime}\right|=|x y|$ and $\left|x^{\prime} y^{\prime \prime}\right|=\left|x^{\prime} y\right|$. Applying the Ptolemy inequality to the quadruple $\left(x, x^{\prime}, y, y^{\prime \prime}\right)$, we have $\left|x^{\prime} y\right| \cdot\left|x y^{\prime \prime}\right|+\left|x^{\prime} y^{\prime \prime}\right| \cdot|x y| \geq\left|x x^{\prime}\right| \cdot\left|y y^{\prime \prime}\right|$. It follows that $2|x y| \cdot\left|x^{\prime} y\right| \geq 2|x y| \cdot\left|x x^{\prime}\right|$. Thus, $\left|x^{\prime} y\right| \geq\left|x x^{\prime}\right|$.

Fix $a>0$ and let $\ell \in X_{\omega}$ be a Ptolemy line. Consider $x, y \in \ell$ such that $|x y|=a / 2$. Let $H_{x}$ and $H_{y}$ be horospheres through $x$ and $y$, and let $\varphi_{x}$ and $\varphi_{y}$ be the symmetries with respect to $H_{x}$ and $H_{y}$. Consider the isometry $\varphi_{y} \circ \varphi_{x}$ and observe that it moves every point along a line Busemann parallel to $\ell$ at the distance $a$. We call such an isometry $a$-shift along $\ell$ and denote it by $\eta_{a, \ell}$. Let $\ell^{\prime}$ be a Ptolemy line (not necessarily Busemann parallel to $\ell$ ). Lemma 5 shows that $\ell^{\prime}$ and $\eta_{a, \ell}\left(\ell^{\prime}\right)$ are Busemann parallel. This means that if $H_{z}$ is the horosphere with respect to $\ell^{\prime}$ through $z$, then $\eta_{a, \ell}\left(H_{z}\right)$ is the horosphere with respect to $\ell^{\prime}$ through $\eta_{a, \ell}(z)$.

6.2. Existence of nonparallel lines. Assume that $X$ is not Möbius equivalent to $\widehat{\mathbb{R}}$.

Lemma 11. For each $\omega, \omega^{\prime} \in X$, there exist distinct Ptolemy lines $\ell, \ell^{\prime} \in X_{\omega}$ such that $\ell \cap \ell^{\prime}=\left\{\omega^{\prime}\right\}$.

Proof. First, we find two Ptolemy circles with exactly two common points. Let $\sigma \subset X$ be a Ptolemy circle, and let $\omega \in \sigma$. Since $X$ is not Möbius equivalent to $\widehat{\mathbb{R}}$, there is $x^{\prime} \in X \backslash \sigma$. Let $c: \mathbb{R} \rightarrow X_{\omega}$ be a unit speed parametrization of the Ptolemy line $\ell=\sigma \backslash \omega$ such that the horosphere $H$ of $\ell$ through $c(0)$ contains $x^{\prime}$. Suppose $z=c(1), z^{\prime}=c(-1)$, and $\left|x^{\prime} z\right|=\left|x^{\prime} z^{\prime}\right|=r$. Consider an s-inversion $\varphi$ with respect to $x^{\prime}, \omega$ and the metric sphere $S_{r}=\left\{x \in X_{\omega}:\left|x^{\prime} x\right|=r\right\}$. By Lemma 2, the image $\varphi(\ell)$ is a Ptolemy circle that intersects $\ell$ at two points $z$ and $z^{\prime}$.

Next let $\sigma_{1}, \sigma_{2}$ be the Ptolemy circles described above, $\sigma_{1} \cap \sigma_{2}=\left\{z, z^{\prime}\right\}$. The lines $\ell_{1, z^{\prime}}=\sigma_{1} \backslash z, \ell_{2, z^{\prime}}=\sigma_{2} \backslash z \subset X_{z}$ through $z^{\prime}$ are not Busemann parallel. Let $\ell_{1, \omega}, \ell_{2, \omega}$ be the lines in $X_{z}$ through $\omega$ that are Busemann parallel to $\ell_{1, z^{\prime}}, \ell_{2, z^{\prime}}$, respectively. Note that $\ell_{1}^{\prime}=\left(\ell_{1, \omega} \backslash\{\omega\}\right) \cup\{z\}$ and $\ell_{2}^{\prime}=\left(\ell_{2, \omega} \backslash\{\omega\}\right) \cup\{z\}$ are Ptolemy lines in $X_{\omega}$. Finally, the Ptolemy lines $\ell_{1}, \ell_{2}$ through $\omega^{\prime}$ Busemann parallel to $\ell_{1}^{\prime}, \ell_{2}^{\prime}$ (respectively) are distinct.

6.3. Homotheties preserve a foliation by horospheres. Suppose $c: \mathbb{R} \rightarrow X_{\omega}$ is a unit speed parametrization of a Ptolemy line $\ell \subset X_{\omega}, o=c(0), z \in \ell$ and $H_{z}$ is the horosphere with respect to $\ell$ through $z$.

Lemma 12. Let $h$ be a homothety with the center o. Then $h\left(H_{z}\right)$ is the horosphere with respect to $\ell$ through $h(z)$.

Proof. Let $x \in H_{z}$, and let $\lambda$ be the coefficient of $h$. Then $\lim _{t \rightarrow \infty}(|x c(t)|-|z c(t)|)=0$. Multiplying by $\lambda$, we have $\lim _{t \rightarrow \infty} \lambda(|x c(t)|-|z c(t)|)=0$. It follows that

$$
\lim _{t \rightarrow \infty}(|h(x) h(c(t))|-|h(z) h(c(t))|)=\lim _{t \rightarrow \infty}(|h(x) c(\lambda t)|-|h(z) c(\lambda t)|)=0,
$$

whence $h\left(H_{z}\right) \subset H_{h(z)}$. On the other hand, for each homothety $h$ we can consider a homothety $h^{\prime}$ with the same center such that $h^{\prime} \circ h=$ id. This means that $h\left(H_{z}\right)=$ $H_{h(z)}$. 
6.4. Projection on horospheres. Here we assume that $X$ is not Möbius equivalent to $\widehat{\mathbb{R}}$. Let $o, \omega \in X$, and let $\ell \subset X_{\omega}$ be a Ptolemy line through $o$.

Suppose $H_{o} \subset X_{\omega}$ is the horosphere with respect to $\ell$ through $o$. We define the projection $\pi_{o}: X_{\omega} \rightarrow H_{o}$ as follows: if $x \in X_{\omega}$ and $\ell_{x}$ is the Ptolemy line through $x$ Busemann parallel to $\ell$, then $\pi_{o}(x):=H_{o} \cap \ell_{x}$.

Proposition 2. Let $\ell^{\prime} \neq \ell \subset X_{\omega}$ be Ptolemy lines through o. Then $\pi_{o}\left(\ell^{\prime}\right)$ is a Ptolemy line.

Proof. We prove that there exists $\alpha>0$ such that $\left|\pi_{o}\left(c^{\prime}(t)\right) \pi_{o}\left(c^{\prime}\left(t^{\prime}\right)\right)\right|=\alpha\left|t-t^{\prime}\right|$ for all $t, t^{\prime} \in \mathbb{R}$, where $c^{\prime}: \mathbb{R} \rightarrow X_{\omega}$ is a unit speed parametrizations of $\ell^{\prime}$ with $c^{\prime}(0)=o$. Put $z=c^{\prime}(1), z^{\prime}=\pi_{o}(z)$, and $\alpha:=\left|o z^{\prime}\right| /|o z|$.

Lemma 13. Let $x_{i}=c^{\prime}\left(t_{i}\right), i=1,2,3$, where $t_{1}<t_{2}<t_{3}$. Then

$$
\frac{\left|\pi_{o}\left(x_{1}\right) \pi_{o}\left(x_{2}\right)\right|}{\left|x_{1} x_{2}\right|}=\frac{\left|\pi_{o}\left(x_{2}\right) \pi_{o}\left(x_{3}\right)\right|}{\left|x_{2} x_{3}\right|}=\frac{\left|\pi_{o}\left(x_{1}\right) \pi_{o}\left(x_{3}\right)\right|}{\left|x_{1} x_{3}\right|} \text {. }
$$

Proof. Let $x_{i} \in \ell_{i}$, where $\ell$ and $\ell_{i}$ are Busemann parallel, and let $x_{i} \in H_{i}$, where $H_{i}$ is the horosphere of $\ell_{i}, i=1,2,3$.

We observe that the homothety $h_{1}: X_{\omega} \rightarrow X_{\omega}$ with the center $x_{1}$ and the coefficient $\left|x_{1} x_{3}\right| /\left|x_{1} x_{2}\right|$ moves $x_{2}$ to $x_{3}$, and $h_{1}\left(H_{1}\right)=H_{1}$. It follows that $h_{1}\left(\ell_{2}\right)=\ell_{3}$. So, if $y_{2}=H_{1} \cap \ell_{2}$ and $y_{3}=H_{1} \cap \ell_{3}$, then $h_{1}\left(y_{2}\right)=y_{3}$. Thus, $\left|x_{1} y_{3}\right| /\left|x_{1} y_{2}\right|=\left|x_{1} x_{3}\right| /\left|x_{1} x_{2}\right|$. On the other hand, $\left|x_{1} y_{3}\right|=\left|\pi_{o}\left(x_{1}\right) \pi_{o}\left(x_{3}\right)\right|$ and $\left|x_{1} y_{2}\right|=\left|\pi_{o}\left(x_{1}\right) \pi_{o}\left(x_{2}\right)\right|$. Consequently,

$$
\frac{\left|\pi_{o}\left(x_{1}\right) \pi_{o}\left(x_{2}\right)\right|}{\left|x_{1} x_{2}\right|}=\frac{\left|\pi_{o}\left(x_{1}\right) \pi_{o}\left(x_{3}\right)\right|}{\left|x_{1} x_{3}\right|} .
$$

In the same way, considering the homothety $h_{3}$ with the center $x_{3}$ and the coefficient $\left|x_{1} x_{3}\right| /\left|x_{2} x_{3}\right|$, we see that

$$
\frac{\left|\pi_{o}\left(x_{2}\right) \pi_{o}\left(x_{3}\right)\right|}{\left|x_{2} x_{3}\right|}=\frac{\left|\pi_{o}\left(x_{1}\right) \pi_{o}\left(x_{3}\right)\right|}{\left|x_{1} x_{3}\right|} .
$$

Now Lemma 13 shows that $\left|\pi_{o}\left(c^{\prime}(t)\right) \pi_{o}\left(c^{\prime}\left(t^{\prime}\right)\right)\right|=\alpha\left|t-t^{\prime}\right|$ for all $t, t^{\prime} \in \mathbb{R}$.

6.5. Horospheres invariance. Let $H_{o} \subset X_{\omega}$ be the horosphere through $o$ with respect to some Ptolemy line $\ell \subset X_{\omega}$.

Proposition 3. The subspace $X^{1}=H_{o} \cup\{\omega\}$ is a compact Ptolemy space with properties (E) and $(\mathrm{sI})$.

Proof. Let $\varphi$ be an s-inversion with respect to $o, \omega \in X$. Note that $\varphi\left(X^{1}\right)=X^{1}$. Indeed, let $z \in H_{o} \cup\{\omega\}$, and let $c: \mathbb{R} \rightarrow X_{\omega}$ be a unit speed parametrization of $\ell$ such that $c(0)=o$. Consider the Busemann function $b: X_{\omega} \rightarrow \mathbb{R}$ of $\ell$ such that $b \circ c(t)=-t$. Then $b(z)=0$. On the other hand, if $z^{\prime}=\varphi(z)$, then

$$
\left|z^{\prime} c(t)\right|=\frac{|z c(1 / t)|}{\frac{1}{t} \cdot|x z|}=\frac{t|z c(1 / t)|}{|x z|} .
$$

Therefore,

$$
b\left(z^{\prime}\right)=\lim _{t \rightarrow \infty}\left(\left|z^{\prime} c(t)\right|-t\right)=\lim _{t \rightarrow \infty}(t|z c(1 / t)| /|x z|-t) .
$$

Note that by $(\nabla)$, we have

$$
|z x|^{2} \leq|z c(1 / t)|^{2} \leq|z x|^{2}+1 / t^{2} .
$$

Then

$$
0 \leq t|z c(1 / t)| /|x z|-t \leq \sqrt{t^{2}+1 /|z x|^{2}}-t .
$$

Thus, $b\left(z^{\prime}\right)=\lim _{t \rightarrow \infty}(t|z c(1 / t)| /|x z|-t)=0$. 
It follows that $\varphi_{x, y}\left(X^{1}\right)=X^{1}$ for any $x, y \in X^{1}$ and any s-inversion $\varphi_{x, y}$ with respect to $x, y$.

Let $x, y \in X^{1}$, and let $S^{\prime} \subset X^{1}$ be a metric sphere between $x$ and $y$ in $X^{1}$. Note that $S^{\prime}=S \cap X^{1}$, where $S \subset X$ is a metric sphere between $x$ and $y$ in $X$. We define an s-inversion $\varphi_{x, y, S^{\prime}}^{\prime}: X^{1} \rightarrow X^{1}$ with respect to $x, y \in X^{1}$ and a metric sphere $S^{\prime} \subset X^{1}$ between $x$ and $y$ as a restriction of an s-inversion $\varphi_{x, y, S}: X \rightarrow X$ with respect to $x, y \in X$ and a metric sphere $S \subset X$ to $X^{1}$. It follows that $X^{1}$ has property (sI).

On the other hand, by Lemma 11] there exists a Ptolemy line $\ell^{\prime} \neq \ell$ through $o$. By Proposition 2, $\pi_{o}\left(\ell^{\prime}\right)$ is a Ptolemy line in $H_{o}$, and then $X^{1}$ has property (E).

6.6. Coordinates in $X_{\omega}$. From now on, we fix $o, \omega \in X$ and consider the metric space $X_{\omega}$. Consider a Ptolemy line $\ell_{0}$ through $o$ with a unit speed parametrization $c_{0}: \mathbb{R} \rightarrow X_{\omega}$, $c_{0}(0)=o$. Let $H_{o}$ be the horosphere with respect to $\ell_{0}$ through $o$, and let $b_{0}: X_{\omega} \rightarrow \mathbb{R}$ be the Busemann function of $\ell_{0}$ with $b_{0}(o)=0$. For each $z \in H_{o}$ denote by $\ell_{z}$ the line Busemann parallel to $\ell_{0}$ through $z$ and consider the unit speed parametrization $c_{z}: \mathbb{R} \rightarrow X_{\omega}$ of $\ell_{z}$ such that $b_{0} \circ c_{0}(t)=-t=b_{0} \circ c_{z}(t)$. From Lemma 4, Corollary 2, and Lemma 8 it follows that the map $i_{1}: \ell_{0} \times H_{o} \rightarrow X_{\omega}$ such that $i_{1}(t, z)=c_{z}(t)$ is a bijection.

Take $x_{0} \in \ell_{0}$ with $\left|o x_{0}\right|=1$. Recall that $\left|z x_{0}\right| \geq\left|o x_{0}\right|=1$ for each $z \in H_{o}$. By Proposition 3 $X^{1}=H_{o} \cup\{\omega\}$ is a compact Ptolemy space with properties (E) and (sI).

Arguing by induction, we obtain a sequence

$$
\cdots \subset X^{k} \subset \cdots \subset X^{1} \subset X^{0}=X
$$

of compact Ptolemy spaces with properties (E) and (sI) and a sequence of points $x_{i} \in$ $X^{i} \backslash X^{i+1}$, where $\left|x_{i} o\right|=1$. Moreover, $\left|x_{i} x_{k}\right| \geq 1$ for $i \neq k$. Since the ball $B_{1}(o)=\{x \in$ $X:|x o| \leq 1\}$ is compact, the sequence $\left\{x_{i}\right\}$ is finite, implying the existence of $N \in \mathbb{N}$ such that $X^{N}$ is Möbius equivalent to $\widehat{\mathbb{R}}$. Then

$$
\widehat{\mathbb{R}}=X^{N} \subset \cdots \subset X^{1} \subset X^{0}=X .
$$

It follows that there is a bijection

$$
i: \ell_{0} \times \ell_{1} \times \cdots \times \ell_{N} \rightarrow X_{\omega} .
$$

This bijection induces on $X_{\omega}$ a structure of the vector space $\mathbb{R}^{N+1}$. This means that we can add points of $X$ and multiply them by real numbers. Note that $o$ plays the role of a neutral element.

Let $b_{i}: X_{\omega} \rightarrow \mathbb{R}, i=1, \ldots, N$, be a Busemann function of $\ell_{i}$ with $b_{i}(o)=0$. Then $b_{i}$ is the $i$ th coordinate function. Moreover, if $H_{i}(x)$ is the horosphere with respect to $b_{i}$ through $x$, then $x=\bigcap_{i=0}^{N} H_{i}(x)$. Denote by $x(i)$ the vector with coordinates $\left(0, \ldots, b_{i}(x), \ldots, 0\right)$, where $b_{i}(x)$ appears at the $i$ th place. Note that $x=x_{0}+\cdots+x_{N}$.

Let $T_{x}^{i}: X_{\omega} \rightarrow X_{\omega}$ be the $b_{i}(x)$-shift along $\ell_{i}$, and let $T_{x}: X_{\omega} \rightarrow X_{\omega}$ be defined by $T_{x}(y)=x+y$ for each $y \in X_{\omega}$. Observe that $T_{x(i)}=T_{x}^{i}$, and then $T_{x}=T_{x}^{N} \circ \cdots \circ T_{x}^{0}$. Consequently, $T_{x}$ is an isometry.

If $h_{k}$ is the homothety with the center $o$ and coefficient $k$, then $h_{k}(x)=k x$, where $k>0$. Indeed, note that $h_{k}(x(i))=k x(i)$. Moreover,

$$
h_{k}\left(H_{i}(x)\right)=h_{k}\left(H_{i}(x(i))=H_{i}(k x(i))=H_{i}(k x)\right.
$$

and

$$
h_{k}(x)=h_{k}\left(\bigcap_{i=0}^{N} H_{i}(x)\right)=\bigcap_{i=0}^{N} H_{i}(k x)=k x .
$$

Therefore, $|o(k x)|=k|o x|$, where $k>0$. 
Let $\nu: X_{\omega} \rightarrow \mathbb{R}_{+}$be defined by $\nu(x)=|o x|$. We prove that $\nu$ is a norm on $X_{\omega}$. Indeed, if $\nu(x)=0$, then $|o x|=0$ and $x=o$. Moreover,

$\nu(x+y)=|o(x+y)| \leq|o x|+|x(x+y)|=|o x|+\left|T_{x}(o) T_{x}(y)\right|=|o x|+|o y|=\nu(x)+\nu(y)$. Finally, observe that $\nu(-x)=|o(-x)|=\left|T_{x}(o) T_{x}(-x)\right|=|x o|=\nu(x)$. So, if $k \geq 0$, then $\nu(k x)=|o(k x)|=k|o x|=k \nu(x)$. If $k<0$, then $\nu(k x)=|o(k x)|=|o(|k|(-x))|=$ $|k||o(-x)|=|k| \nu(-x)=|k| \nu(x)$.

Also we note that $\nu(\cdot)$ induces the metric of $X_{\omega}$. Indeed, $|x y|=\left|T_{x}(o) T_{x}(y-x)\right|=$ $\nu(y-x)$. Applying the Schoenberg theorem, see Theorem 2 we conclude that $X$ is Möbius equivalent to $\widehat{\mathbb{R}}^{N}$.

\section{ACKnowledgements}

I would like to thank Sergei Buyalo for valuable advice and attention to this paper.

\section{REFERENCES}

[BS1] S. Buyalo and V. Schroeder, Moebius structures and Ptolemy spaces: boundary at infinity of complex hyperbolic spaces, arXiv:1012.1699.

[BS2] _ Moebius characterization of the boundary at infinity of rank one symmetric spaces, arXiv: 1211.3237.

[FS] Th. Foertsch and V. Schroeder, Metric Möbius geometry and a characterization of spheres, Manuscripta Math. 140 (2013), no. 3-4, 613-620. MR3019142

[Sch] I. Schoenberg, A remark on M. M. Day's characterization of inner-product spaces and a conjecture of L. M. Blumenthal, Proc. Amer. Math. Soc. 3 (1952), 961-964. MR0052035(14:564c)

St. Petersburg Branch, Steklov Mathematical Institute, Russian Academy of Sciences, Fontanka 27, Saint Petersburg 191023, Russia

E-mail address: alvismi@gmail.com

Received 23/JAN/2013

Translated by THE AUTHOR 